\title{
Surface structuring of fused silica with asymmetric femtosecond laser pulse bursts
}

\author{
Javier Hernandez-Rueda, ${ }^{1,2}$ Jan Siegel, ${ }^{1,3}$ Marcial Galvan-Sosa, ${ }^{1}$ Alexandro Ruiz de la Cruz, ${ }^{1}$ \\ and Javier Solis ${ }^{1}$ \\ ${ }^{1}$ Laser Processing Group, Instituto de Óptica, C.S.I.C., Serrano 121, Madrid E-28006, Spain \\ ${ }^{2}$ e-mail: javihr@io.cfmac.csic.es \\ ${ }^{3}$ e-mail: j.siegel@io.cfmac.csic.es
}

Received March 4, 2013; accepted March 19, 2013;

posted April 2, 2013 (Doc. ID 186286); published April 24, 2013

\begin{abstract}
Fused silica surface structuring has been performed using temporally shaped femtosecond laser pulses. For this purpose we have designed pulse bursts with a triangular intensity envelope and different slope sign and interpulse separation that were experimentally generated using a home-made temporal pulse shaper. We have found that pulse bursts with decreasing intensity envelopes are remarkably more efficient in terms of surface ablation than bursts with increasing intensity envelopes. The results reveal that laser energy coupling in the material is enhanced as the interpulse spacing decreases. A study of the ablation depth using stretched single pulses was carried out and compared to results obtained for pulse bursts with different interpulse spacing. We find that the deepest crater was achieved with bursts of $0.5 \mathrm{ps}$ interpulse separation and decreasing envelope. This pulse form also induced the largest change of the surface reflectivity after irradiation. The results are discussed in terms of how the laser energy coupling efficiency is linked to the temporal pulse shape. ( 2013 Optical Society of America
\end{abstract}

OCIS codes: (320.5540) Pulse shaping; (320.7120) Ultrafast phenomena.

http://dx.doi.org/10.1364/JOSAB.30.001352

\section{INTRODUCTION}

The last two decades have witnessed the rise of nonlinear laser processing of dielectric materials, which makes possible, among other applications, the fabrication of integrated photonic devices by direct laser writing $[1,2]$. The fabrication of surface and bulk elements with submicrometer resolution is feasible by using ultrashort laser pulses because the excitation and relaxation channels result in minimal collateral damage $[\underline{3}, \underline{4}]$. Morphology of single-pulse or multipulse generated structures, though, requires optimization in terms of feature size, roughness, and so forth in order to obtain high-quality structures for micro- and nanoscale applications $[\underline{5,}, 6]$. In this respect, it is worth noticing that the morphology and ablation depth are directly related to the ionization mechanisms involved. Much effort has been devoted to analyzing such a relation by modeling free electron generation via multiphoton and avalanche ionization mechanisms, in particular using methods based on multiple rate equations [구, $\underline{\text { ] }}$. Experimentally, the plasma dynamics in dielectrics have been investigated, by studying the temporal evolution of the optical properties of the irradiated region [9-11] and by means of spectral interference methods [12], but the relative contributions of the individual ionization processes, as well as the laser temporal profile dependences, are still an active subject of study. Over the last years the use of temporally shaped ultrashort laser pulses has been exploited in order to investigate and control the laser energy coupling into the dielectric material for a variety of temporal shapes [13-16]. Some of these works were carried out spreading the energy of the laser in bursts of pulses by exploring third-order spectral phase values [13]. Other investigations employed double or multiple pulses [11] along with adaptive methods using genetic algorithms and a feedback signal or Gerchberg-Saxton algorithms [15].

In this paper we report results on topography and optical property changes upon irradiation with temporally shaped ultrashort laser pulses in fused silica. We have performed a systematic study of irradiations using bursts of four pulses with different interpulse separations for burst intensity envelopes either decreasing or increasing. The topography changes induced are correlated to those induced by single stretched pulses and to changes in the optical reflectivity of the sample upon irradiation.

\section{EXPERIMENTAL}

The laser source used in this work is a Ti:sapphire ultrafast regenerative amplifier (Spitfire-Pro, Spectra-Physics), which delivers transform-limited pulses of 100 fs pulse duration centered at $800 \mathrm{~nm}$ wavelength and $1 \mathrm{~mJ}$ pulse energy. For pulse shaping we used a home-built temporal pulse shaper based on the layout described by Wollenhaupt et al. [17] and Weiner [18]. Briefly, it makes use of a liquid crystal spatial light modulator (LC-SLM-S320, Jenoptik) with a zero dispersion system based on the $4 f$ system [19]. The $4 f$ system consists of two diffraction gratings in Littrow configuration and a pair of cylindrical mirrors with the same focal length $(f=258 \mathrm{~mm})$ to collimate the dispersed wavelength components. The spatial light modulator is placed in the Fourier plane between the mirrors and consists of a one-dimensional array of 320 pixels. Each pixel has an effective width of $97 \mu \mathrm{m}$ and allows modifying the spectral phase via orientation of liquid crystal molecules, with a spectral resolution of $0.11 \mathrm{~nm} /$ pixel. The 
spectral phase of the shaped pulses is calculated using a program based on a Gerchberg-Saxton algorithm and a fast Fourier transformation, where the pulse temporal energy distribution can be designed at wish. For the present study we have chosen bursts of four pulses, controlling their relative amplitude, along with the temporal separation. The irradiations performed with transform-limited and linearly chirped stretched pulses were carried out without passing through the shaper and simply detuning the compressor stage of the femtosecond laser amplifier. For linearly chirped pulses we used $500 \mathrm{fs}$ pulse duration as this time range is near the range of the maximum electron plasma absorption investigated in previous works [16].

For the laser pulse characterization we have used a homemade frequency-resolved optical gating system in polarization gate (PG-FROG) configuration, as described by Trebino et al. [20]. The PG configuration has the advantage of obtaining the temporal structure of the pulse with no ambiguities in the time-axis direction. The layout of the PG-FROG setup was designed in a way to enable measuring shaped pulses with long temporal pulse shapes up to tens of picoseconds, as well as relatively low peak powers. The latter was achieved by using a material with high third-order optical susceptibility (SF57, Schott) as the nonlinear medium in the PG-FROG setup.

For the experiments using pulse bursts a single transformlimited pulse of $100 \mathrm{fs}$ duration is shaped using the temporal pulse-shaper apparatus. Afterwards, the shaped pulse is sent through a half-wave plate combined with a polarizing cube beam splitter to adjust the laser pulse energy, which is measured using a photodiode previously calibrated by means of a powermeter. Then the $s$-polarized pump pulse is focused with a lens $(f=50 \mathrm{~mm})$ on the sample surface at an angle of $54^{\circ}$ to a Gaussian spot size $\left(1 / e^{2}\right.$ diameter $)$ of $16.9 \mu \mathrm{m} \times 8.0 \mu \mathrm{m}$ for the pulse bursts and $9.5 \mu \mathrm{m} \times 4.5 \mu \mathrm{m}$ for the transform-limited and stretched pulses, leading to a maximum local fluence up to $11.8 \mathrm{~J} / \mathrm{cm}^{2}$. The spot size is determined by fitting the diameter of the ablated crater versus pulse energy employing a standard Gaussian formula, as described by Liu [21].

The sample surface is monitored during the process of irradiation by means of a wide-field microscope consisting of a microscope objective (Mitutoyo, $80 \times, \mathrm{NA}=0.50$ ), a tube lens $(f=200 \mathrm{~mm})$, a 12 bit charge-coupled device (CCD), and an LED (400 nm) to illuminate the sample. The study was carried out in fused silica (Lithosil samples by Schott), and each area was irradiated only once, moving the sample to a fresh region before irradiation. The sample was cleaned before and after irradiation using a process with organic and inorganic chemical solvents with an ultrasonic bath [22].

After sample irradiation and cleaning, the surface morphology was studied using different techniques in order to investigate the influence of the pulse shape on the material response. The topography characterization of the irradiated areas was performed using an interferometric microscope (Sensofar profiler at $460 \mathrm{~nm}, \mathrm{NA}=0.55$, objective $50 \times$ ) with a depth resolution of $\sim 1 \mathrm{~nm}$. The reflectivity of the sample surface was studied with an optical microscope in reflection mode using two different monochromatic light sources (Nikon Eclipse at 460 and $850 \mathrm{~nm}, \mathrm{NA}=0.9$, objective $100 \times$ ). In order to investigate the crater morphology in more detail we carried out scanning electron microscopy (SEM) observations. For this purpose the sample was coated with a $5 \mathrm{~nm} \mathrm{Au}$ thin film in order to avoid space charge effects.

\section{RESULTS AND DISCUSSION}

Figure 1 shows a set of measurements of the crater crosssection profiles produced with different pulse shapes. The corresponding temporal profiles (retrieved from the PG-FROG traces) are also included. Irradiations in Fig. 1 were performed at a fluence of $F \approx 6.0 \pm 0.5 \mathrm{~J} / \mathrm{cm}^{2}$. Figures $\underline{1(\mathrm{a})}$ and $1(\mathrm{~b})$ show the results obtained using a transform-limited pulse of $100 \mathrm{fs}$ and a stretched pulse of 500 fs pulse duration, respectively. For the same fluence the shorter pulse generates a crater with a depth $d \sim 100 \mathrm{~nm}$ whereas the stretched pulse produces a shallower crater with $d \sim 55 \mathrm{~nm}$. Figures $\underline{1(\mathrm{c})}-\underline{1(\mathrm{j})}$ show the results obtained with bursts of pulses with separations from 2 ps down to 0.5 ps between consecutive pulses. While none of the profiles produces a deeper crater than the one achieved with a transform-limited pulse, some pulse structures produce craters significantly deeper than the one produced by the 500 fs stretched pulse. It can be seen that the crater depth increases as the burst spacing decreases (e.g., $2 \mathrm{ps}^{-}$and $0.8 \mathrm{ps}^{-}$). It is also remarkable that the sign of the burst intensity envelope has a strong influence on the crater depth. For all pulse spacings a decreasing envelope (e.g., $2 \mathrm{ps}^{-}$) is more efficient than an increasing one (e.g., $2 \mathrm{ps}^{+}$). The underlying reason is that for an increasing envelope the energy of the first pulse is not fully coupled to the material due to the lower peak intensity, which reduces the effectiveness of multiphoton absorption. An overall comparison of the pulse train forms explored reveals that bursts of pulses with a decreasing envelope and a spacing of $0.5 \mathrm{ps}$ are the most favorable to maximize the depth efficiency of the ablation process. Also, this pulse form produces a deeper crater than using a stretched pulse with a 500 fs pulse duration.

Figure 2 shows the crater depth as a function of laser fluence using bursts of pulses with spacings ranging from 0.5 to 2 ps. While for 2 and 1 ps spacings only a little influence of the

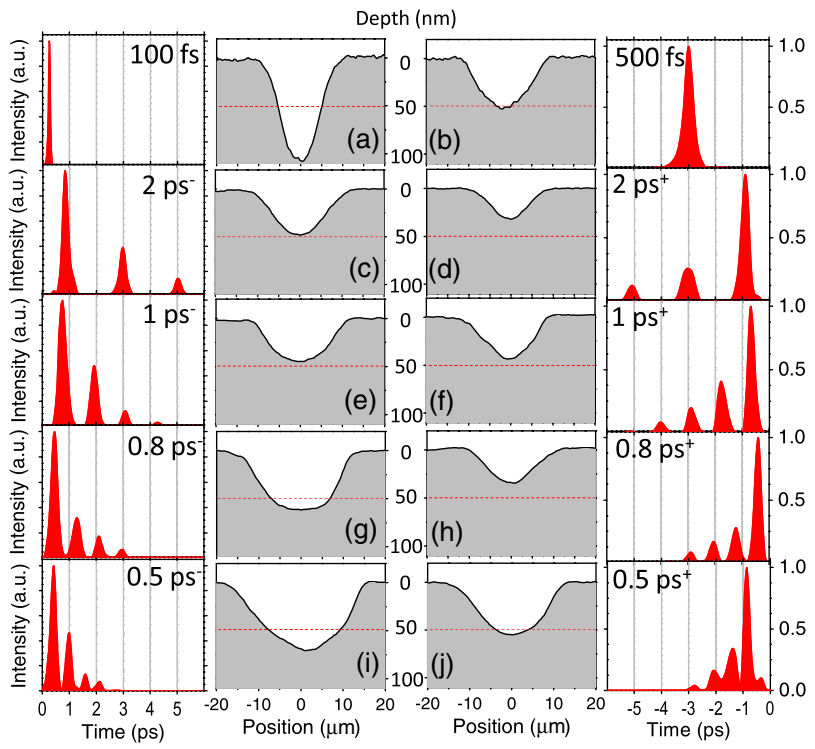

Fig. 1. Experimentally measured pulse shapes and crater depth profiles induced for each pulse at fluence of $F=6.0 \pm 0.5 \mathrm{~J} / \mathrm{cm}^{2}$. The red line marks a $50 \mathrm{~nm}$ crater depth. 


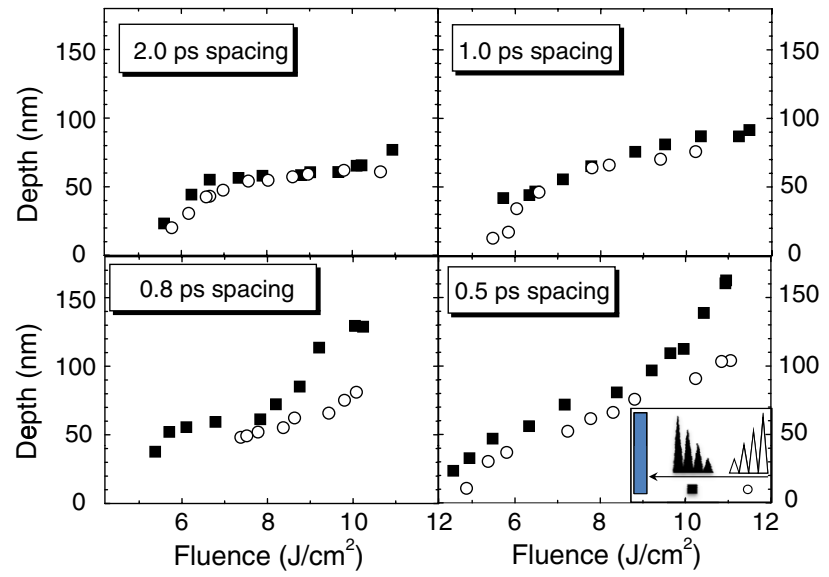

Fig. 2. Crater depth as a function of laser fluence for pulse bursts with different interpulse spacing. The inset illustrates the sign of the bursts; circles correspond to increasing envelope, squares to decreasing envelope.

envelope sign on the ablation behavior is observed, for 0.8 and $0.5 \mathrm{ps}$ spacings, a clear increase in ablation efficiency for bursts with decreasing envelope can be observed. Moreover, in agreement with the tendency shown in Fig. 1, bursts with decreasing envelope and 0.5 ps spacing produce the deepest crater with $d=163 \mathrm{~nm}$ at maximum fluence. The results can be understood taking into account the transient changes in the energy coupling efficiency for the different pulses as they interact with electron plasmas with different carrier densities as a direct consequence of the burst spacing. The first pulse generates a dense electron plasma via multiphoton ionization, whereas subsequent pulses are more or less efficiently absorbed depending on the density of the plasma initially induced and their relative delay. As shown in [10], the plasma formation, upon $100 \mathrm{fs}$ pulses, leads to a strong increase of the absorption within $500 \mathrm{fs}$ and a somewhat slower increase of the reflectivity within 1-2 ps. For temporal values $t>1 \mathrm{ps}$ the second pulse is therefore partially reflected due to the increase of the transient reflectivity. In contrast, for an interpulse spacing of $t<1$ ps the second pulse is more efficiently absorbed, consistent with the fact that by using bursts with 0.5 ps spacing the deepest crater is produced [23].

We have also performed a study of the ablation depth as a function of interpulse spacing for bursts and of pulse duration for single pulses at two constant fluence values, the results being shown in Fig. 3. At $9.8 \mathrm{~J} / \mathrm{cm}^{2}$ a crater depth of $d=$ $180 \mathrm{~nm}$ is achieved for a transform-limited pulse. The depth

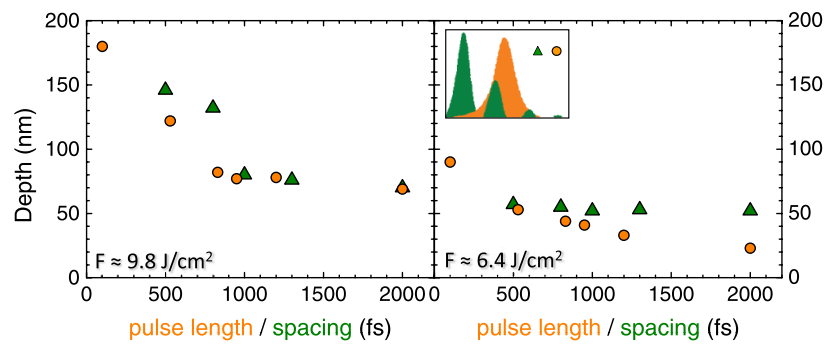

Fig. 3. Crater depth as a function of pulse duration for single pulses and interpulse spacing for bursts at a fluence of (a) $9.8 \mathrm{~J} / \mathrm{cm}^{2}$ and (b) $6.4 \mathrm{~J} / \mathrm{cm}^{2}$. The inset in (b) shows a schematic representation of the temporal intensity distribution; triangles correspond to pulse bursts and circles to single pulses. then decreases gradually upon pulse stretching down to $d=$ $70 \mathrm{~nm}$ at $1 \mathrm{ps}$ pulse duration. Craters produced by pulse bursts with interpulse spacings of 0.5 and $0.8 \mathrm{ps}$ are deeper than those produced with stretched pulses. This fact can be understood taking into account the temporal structure of the pulse, in particular the peak intensity of the different temporal pulse shapes. While the integrated energy is the same for each laser pulse shape, it is more efficient to concentrate the energy in four light packets with an interpulse spacing in the subpicosecond range and a moderate peak intensity than to spread it over a single stretched pulse intensity profile, leading to a relatively low peak intensity. For wider interpulse spacings $(>0.8 \mathrm{ps})$ the ablation depth is the same as for stretched pulses. At moderate fluences $\left(6.4 \mathrm{~J} / \mathrm{cm}^{2}\right)$ the depth achieved is nearly constant for pulse bursts, while it decreases with pulse duration for stretched pulses. The former can be explained by their relatively low fluence, which causes the pulses after the first one to be too weak to significantly contribute to the ablation process.

Figure $\underline{4}$ links the findings about crater depth with the corresponding changes in the reflectivity of the irradiated region by comparing optical microscopy images and topography maps. The reflectivity images were measured using an optical microscope in reflection mode with a monochromatic light source centered at $460 \mathrm{~nm}$. The spatial extension of reflectivity changes matches well that of topography changes for all pulse forms. Moreover, the chosen representation allows relating certain reflectivity values with specific depth values by means of the false color scale. The best way to relate them
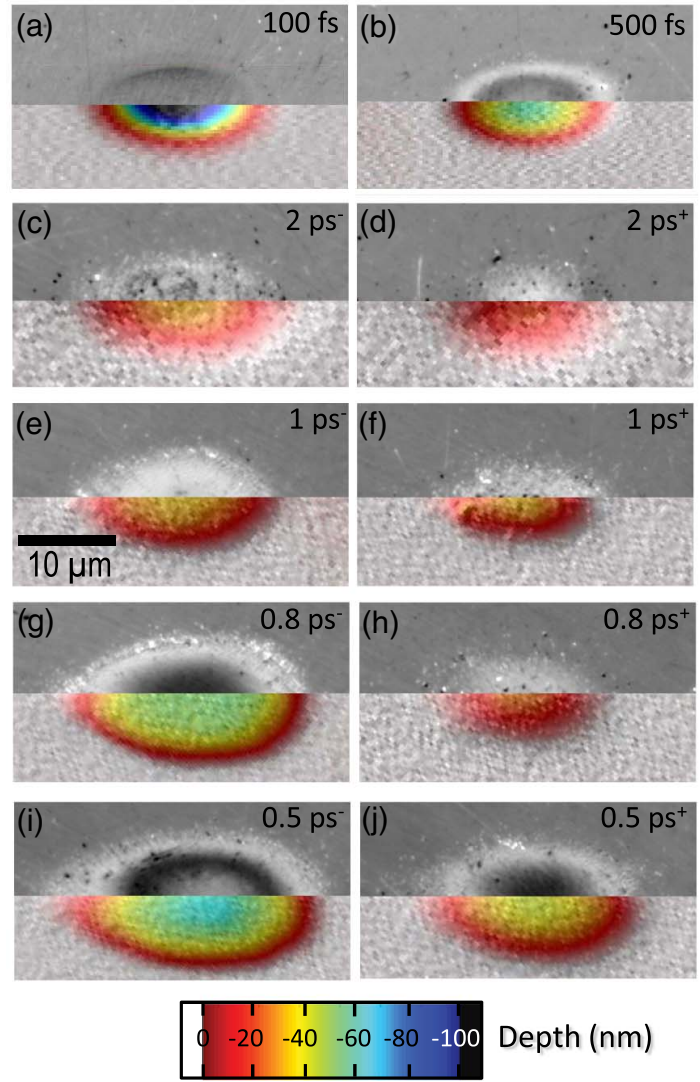

Fig. 4. (a)-(j) Combined graphs of microscopy images (upper half) and topography maps (lower half). The topography micrographs were measured using an interferometric microscope. 

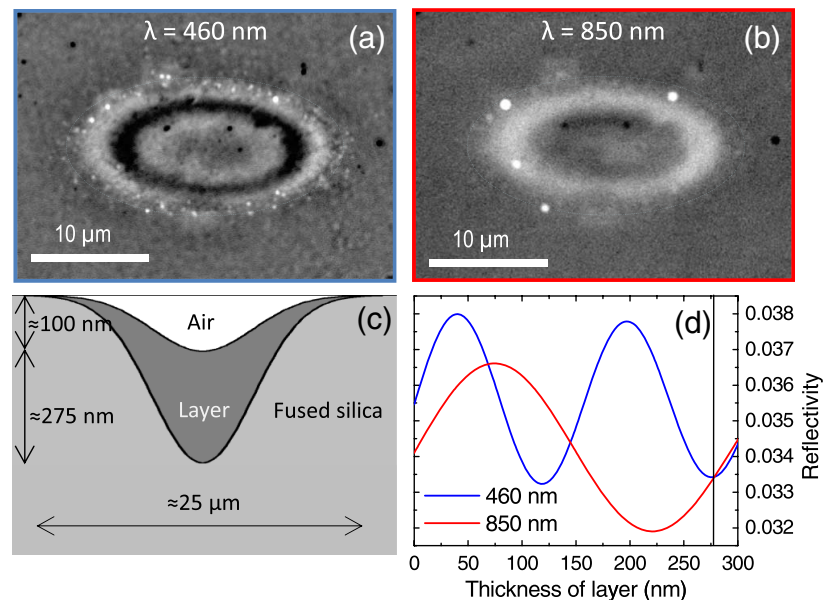

Fig. 5. (a), (b) Optical microscopy images of the same crater produced using a $500 \mathrm{fs}$ single laser pulse at $F \sim 9.1 \mathrm{~J} / \mathrm{cm}^{2}$, illuminated with monochromatic light of 460 and $850 \mathrm{~nm}$, respectively. (c) Scheme of a micro-Fabry-Perot system as result of the laser-induced layer underneath the ablation crater. (d) Calculated reflectivity modulation using an increased absorption of $\kappa=0.02$ for the laser-induced layer.

is using the $0.5 \mathrm{ps}^{-}$data [Fig. $\underline{4(\mathrm{i})}$ ] as an example because it allows accessing all reflectivity and depth regimes. The bright outer ring in the reflectivity image features a reflectivity increase of $\Delta R^{+} / R o=+7 \%$, which corresponds to depths up to approximately $35 \mathrm{~nm}$. The next inner black ring shows a reflectivity decrease of $\Delta R / R o=-7 \%$ and corresponds to a depth range from 45 to $65 \mathrm{~nm}$, and the inner grey disc reaches a maximum depth of $75 \mathrm{~nm}$. We have found this relation to be valid for irradiations produced by all other pulse forms except for transform-limited $100 \mathrm{fs}$ pulses. In this last case, the reflectivity increase in the outer ring with a maximum depth of $30 \mathrm{~nm}$ is much less, although still present [24].

The appearance of dark and bright rings with a considerable optical contrast suggests the presence of interference phenomena. In order to investigate their origin we have performed optical microscopy observations using monochromatic light sources with two different wavelengths. Figure $\underline{5}$ shows the very same region irradiated with a single laser pulse of $500 \mathrm{fs}$ FWHM at a fluence of $F=9.1 \mathrm{~J} / \mathrm{cm}^{2}$ illuminated with 460 and $850 \mathrm{~nm}$. Both micrographs show a ring pattern, although for the one measured with $460 \mathrm{~nm}$ light the number of fringes is approximately twice that for the one measured using the $850 \mathrm{~nm}$ source. This fact indicates that the ratio between the number of fringes and wavelength is approximately constant, verifying the interferometric origin for the fringe pattern, corresponding to Newton fringes caused by a semitransparent, superficially modified surface layer. We previously observed and studied this kind of interference effect for irradiations in phosphate glass, using 100 fs transformlimited pulses [25]. In the current investigation, we modeled the ring pattern observation by analogy with a micro-FabryPerot system, a laser-induced thin surface layer with different optical properties as sketched in Fig. 5(c). The simulation results suggest an increase of the local absorption of $\Delta \kappa \approx 0.02$ but no change in the real part of the refractive index. Using these values for the optical properties, the simulations lead to a reflectivity amplitude of $\Delta R / R o= \pm 0.07$ for the Newton rings studied with $460 \mathrm{~nm}$ light and $\Delta R / R o= \pm 0.04$ for the pattern studied with $850 \mathrm{~nm}$ light [Fig. $5(\mathrm{~d})$ ], consistent with the experimental data [Figs. 5(a) and $5 \overline{(\mathrm{b})}$ ].

Finally, in order to study if other material modifications, with a different local absorption increase, contribute to the reflectivity changes produced by stretched and shaped pulses, we have measured the irradiated areas with SEM. Figure $\underline{6}$ shows SEM micrographs combined with optical microscopy images recorded for irradiations using 100 fs transformlimited pulses [Fig. 6(a)], 500 fs stretched pulses [Fig. 6(b)], and a pulse burst with 0.8 ps interpulse spacing [Fig. $\underline{6(\mathrm{c})}$ ] The second row shows an overview of the whole crater using SEM, and the third row shows a zoomed area, indicated by a white rectangle, of the crater profile. In general shaped pulses feature a moderate surface roughness for each pulse structure whereas the one produced by using 100 fs transform-limited pulses appears to be smoother. This roughness on the edge of the crater produced by these kinds of shaped and stretched pulses is characteristic of long temporal pulses. This sort of morphology might be produced because of the different evolution through the ionization mechanisms, whose contribution is weighted depending on the pulse duration by balancing the multiphoton ionization and avalanche ionization. This fact produces a relatively high surface roughness compared with the smoothness produced for shorter pulses [6,14]. The microscopy images in the first row show the spatial correspondence between the characteristic surface roughness
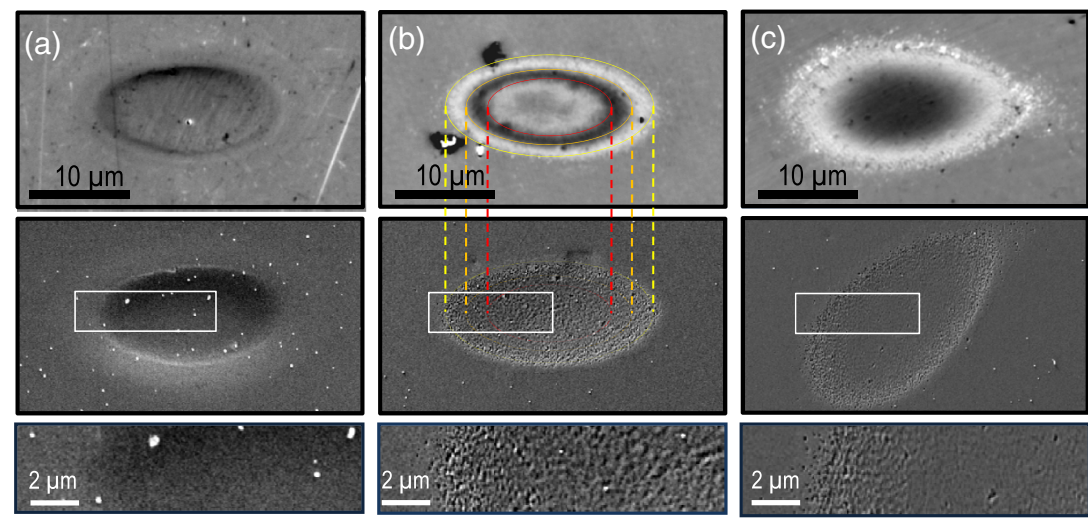

Fig. 6. (a)-(c) SEM images (second and third rows) of the irradiated area measured using secondary electrons detector and optical microscopy images (first row). The irradiations were performed using a single Gaussian pulse of (a) 100 fs duration at $F \sim 9.1 \mathrm{~J} / \mathrm{cm}^{2}$, (b) $500 \mathrm{fs}$ duration, and (c) a burst of pulses with negative envelope and an interpulse spacing of $0.8 \mathrm{ps}$. The small white rectangles in the second row SEM images highlight the crater profile morphology shown on the third row. 
and the modulation of the surface reflectivity. This comparison between each area studied with SEM and optical microscopy confirms that the Newton rings are not induced by roughness because the reflectivity pattern does not match the roughness pattern. Given the size of the roughness features (from tens to hundreds of nanometers), we can expect that roughness will only affect the overall visibility of the Newton rings via scattering.

\section{CONCLUSION}

We have investigated the influence of the pulse shape on the ablation depth, optical reflectivity, and surface morphology in fused silica irradiated with temporally shaped femtosecond laser pulses. The results clearly indicate that the way the laser energy is distributed over time has a strong impact on the efficiency of material removal due to inherent changes in the laser energy coupling in the material. High ablation efficiency using pulse bursts requires simultaneously (1) an initially sufficient amount of multiphoton absorption and (2) the generation of a moderate plasma density in order that subsequent pulses can be effectively absorbed by the previously created carrier plasma (i.e., a plasma density too high would render a larger plasma reflectivity). When comparing the material behavior upon irradiation with stretched pulses, the results also show that it is clearly more efficient to concentrate the total energy of the pulses in several energy packets with moderate peak power than to spread it over a relatively longer single pulse.

\section{ACKNOWLEDGMENTS}

This work has been partially supported by the Spanish TEC2011-22422 project. J. H.-R. acknowledges a grant awarded by the Spanish Ministry of Science and Innovation. We are grateful to Professor S. Marcos and P. Pérez from IO-CSIC for providing access and assistance in optical interferometer measurements. We acknowledge Professor P. Leiderer and B. Kopp from Konstanz University and Professor C. Molpeceres and S. Lauzurica from Universidad Politécnica for SEM measurements.

\section{REFERENCES AND NOTES}

1. H. Misawa and S. Juodkazis, 3D Laser Microfabrication (Wiley-VcH Verlag, 2006).

2. K. Sugioka, M. Meunier, and A. Piqué, Laser Precision Micromachining (Springer-Heidelberg, 2010)

3. A. P. Joglekar, H. Liu, E. Meyhöfer, G. Mourou, and A. J. Hunt, "Optics at critical intensity: applications to nanomorphing," Proc. Natl. Acad. Sci. U.S.A. 101, 5856-5861 (2004).

4. G. Miyaji and K. Miyazaki, "Origin of periodicity in nanostructuring on thin film surfaces ablated with femtosecond laser pulses," Opt. Express 16, 16265-16271 (2008).

5. D. Ashkenasi, R. Stoian, A. Rosenfeld, and E. E. B. Campbell, "Coulomb explosion in ultrashort pulsed laser ablation of $\mathrm{Al}_{2} \mathrm{O}_{3}$," Phys. Rev. B 62, 13167-13173 (2000).

6. F. Watanabe, D. G. Cahill, B. Gundrum, and R. S. Averback, "Ablation of crystalline oxides by infrared femtosecond laser pulses," J. Appl. Phys. 100, 083519 (2006).

7. B. Rethfeld, "Free-electron generation in laser-irradiated dielectrics," Phys. Rev. B 73, 035101 (2006).

8. P. Balling and J. Schou, "Femtosecond-laser ablation dynamics of dielectrics: basics and applications for thin films," Rep. Prog. Phys. 76, 036502 (2013).
9. J. Siegel, D. Puerto, W. Gawelda, G. Bachelier, J. Solis, L. Ehrentraut, and J. Bonse, "Plasma formation and structural modification below the visible ablation threshold in fused silica upon femtosecond laser irradiation," Appl. Phys. Lett. 91, 082902 (2007).

10. J. Hernandez-Rueda, D. Puerto, J. Siegel, M. Galvan-Sosa, and J. Solis, "Plasma dynamics and structural modifications induced by femtosecond laser pulses in quartz," Appl. Surf. Sci. 258 9389-9393 (2012).

11. R. Stoian, A. Mermillod-Blondin, S. W. Winkler, A. Rosenfeld, I. V. Hertel, M. Spyridaki, E. Koudoumas, P. Tzanetakis, C. Fotakis, I. M. Burakov, and N. M. Bulgakova, "Temporal pulse manipulation and consequences for ultrafast laser processing of materials," Opt. Eng. 44, 051106 (2005).

12. C. Sarpe, J. Köhler, T. Winkler, M. Wollenhaupt, and T. Baumert, "Real-time observation of transient electron density in water irradiated with tailored femtosecond laser pulses," New J. Phys. 14, 075021 (2012).

13. L. Englert, B. Rethfeld, L. Haag, M. Wollenhaupt, C. SarpeTudoran, and T. Baumert, "Control of ionization processes in high band gap materials via tailored femtosecond pulses," Opt. Express 15, 17855-17862 (2007).

14. M. Boyle, R. Stoian, A. Thoss, A. Rosenfeld, G. Korn, and I. V. Hertel, "Laser ablation of dielectrics with temporally shaped femtosecond pulses," Appl. Phys. Lett. 80, 353 (2002).

15. R. Stoian, M. Wollenhaupt, T. Baumert, and I. V. Hertel, "Tempoal pulse tailoring in ultrafast laser manufacturing technologies," in Laser Precision Microfabrication, Springer Series in Material Sciences 135 (Springer, 2010), pp. 121-144.

16. J. Hernandez-Rueda, J. Siegel, D. Puerto, M. Galvan-Sosa, W. Gawelda, and J. Solis, "Ad-hoc design of temporally shaped fs laser pulses based on plasma dynamics for deep ablation in fused silica," Appl. Phys. A, doi: 10.1007/s00339-012-7238-2 (2012).

17. M. Wollenhaupt, A. Präkelt, A. Assion, C. Horn, C. SarpeTudoran, M. Winter, and T. Baumert, "Compact, robust, and flexible setup for femtosecond pulse shaping,” Rev. Sci. Instrum. 74, 4950 (2003).

18. A. M. Weiner, "Femtosecond pulse shaping using spatial light modulators," Rev. Sci. Instrum. 71, 1929 (2000).

19. O. E. Martinez, "Matrix formalism for pulse compressors," IEEE J. Quantum Electron. 24, 2530-2536 (1988).

20. R. Trebino, K. W. DeLong, D. N. Fittinghoff, J. N. Sweetser, M. A. Krumbügel, B. A. Richman, and D. J. Kane, "Measuring ultrashort laser pulses in the time-frequency domain using frequency-resolved optical gating," Rev. Sci. Instrum. 68, 3277 (1997).

21. J. M. Liu, "Simple technique for measurements of pulsed Gaussian-beam spot sizes," Opt. Lett. 7, 196-198 (1982).

22. L. Jiao, Y. Jin, Y. Ji, Y. Tong, F. Wang, T. Liu, and L. Wang, "Research on chemical cleaning technology for super-smooth surface of fused silica substrate," Proc. SPIE 7655, 76552J (2010).

23. M. Lebugle, N. Sanner, S. Pierrot, and O. Utéza, "Absorption dynamics of a femtosecond laser pulse at the surface of dielectrics," AIP Conf. Proc. 1464, 91-101 (2012).

24. For transform-limited pulses the relation between permanent optical changes and change of surface topography was studied in previous works $[9,10]$. By means of femtosecond microscopy, topography measurements, and conventional microscopy, it was concluded that the annular topography change of $30 \mathrm{~nm}$ corresponds to a surface depression via densification, which leads to an increase in reflectivity caused by an increase of the refractive index. For transform-limited pulses the reflectivity increment $\Delta R / R o \approx 3 \%$, and the corresponding structural change leads to an increment of refractive index produced by density increase $\Delta n / n o \approx \Delta \rho / \rho o \approx 0.6 \%$.

25. D. Puerto, J. Siegel, A. Ferrer, J. Hernandez-Rueda, and J. Solis, "Correlation of the refractive index change at the surface and inside phosphate glass upon femtosecond laser irradiation," J. Opt. Soc. Am. B 29, 2665-2668 (2012). 